\title{
Neurologic sequelae associated with delayed identification of iatrogenic skull base injury during endoscopic sinus surgery $(E S S)^{*}$
}

Mark Kubik, Stella Lee, Carl Snyderman, Eric Wang

Center for Cranial Base Surgery, Department of Otolaryngology, University of Pittsburgh, Pittsburgh, PA, USA
Rhinology 55: 53-58, 2017

https://doi.org/10.4193/Rhino16.169

*Received for publication:

May 24, 2016

Accepted: August 23, 2016

\begin{abstract}
Background: Skull base injury is an infrequent complication during endoscopic sinus surgery (ESS). We hypothesize that late recognition and repair of CSF leaks during ESS is associated with increased neurologic morbidity.
\end{abstract}

Methodology: A retrospective review was performed of patients with skull base injury during ESS at a tertiary center from 19992015. The study population was separated into early ( $<72 \mathrm{hrs}$ ) and late ( $>72 \mathrm{hrs}$ ) intervention groups. Study parameters included time to diagnosis, imaging, site of injury, type of repair, neurologic complications, and clinical outcomes.

Results: Seventeen patients were studied. The primary surgical interventions included ESS and balloon sinuplasty. Mean latency from primary surgery to presentation to our facility was 11 days. The most common injury site was the cribriform plate and the mean defect size $4.5 \mathrm{~mm}$. Late diagnosis was associated with increased total neurologic complications and increased rates of postoperative meningitis. No neurologic complications occurred in patients diagnosed intraoperatively. All patients underwent successful endoscopic repair.

Conclusion: Skull base injury is a rare but major complication following both balloon sinuplasty and traditional primary ESS. Early diagnosis and endoscopic repair may prevent neurologic morbidity in these patients.

Key words: skull base injury, CSF, endoscopic sinus surgery, delayed diagnosis

\section{Introduction}

Endoscopic sinus surgery (ESS) is the treatment of choice for chronic rhinosinusitis refractory to comprehensive medical management ${ }^{(1)}$. As a treatment modality, ESS is becoming increasingly utilized and today over 600,000 cases are performed in the United States annually ${ }^{(2)}$. Major complications after ESS are rare and occur in $<1 \%$ of cases ${ }^{(3-11)}$. These generally include orbital injury, major vascular injury, and skull base injury with resultant cerebrospinal fluid (CSF) rhinorrhea. Risk factors for complications include anatomic variants, revision surgery, disease burden, nasal polyposis, and use of powered instrumentation ${ }^{(3-6,12-13)}$.

The incidence of CSF rhinorrhea following ESS is exceedingly low and has been estimated in several large retrospective series to be $0.13-0.5 \%{ }^{(3-7)}$. Although rare, skull base transgression during ESS is associated with significant morbidity and mortality. Complications can include CSF rhinorrhea with ascending meningitis, major intracranial vascular injury, subarachnoid hemorrhage, frontal lobe injury, and postoperative pneumocephalus ${ }^{(8)}$.

The management of skull base injury during ESS includes the timely recogencnition of the injury, clinical and imaging evaluation for intracranial complications, and surgical repair ${ }^{(14)}$. Clinical experience suggests that conservative, nonsurgical management of iatrogenic CSF rhinorrhea generally fails ${ }^{(15)}$. While surgical repair historically involved transcranial and external approaches ${ }^{(16)}$, endoscopic endonasal techniques are well described and associated with success rates of $>96 \%$, establishing this technique as the standard of care ${ }^{(17-27)}$. However, delays in the diagnosis and management of this complication has not been 
investigated. In maxillofacial and anterior skull base trauma, early diagnosis and management of CSF rhinorrhea has been associated with reduced neurologic morbidity, specifically with regard to the prevention of ascending meningitis(28). Given the rarity of iatrogenic skull base injuries in ESS, the relationship between the latency to diagnosis and the subsequent neurologic morbidity is unclear.

The primary objective of this study is to compare the associated neurologic morbidity between early and delayed interventions in patients diagnosed with iatrogenic CSF leaks from ESS.

\section{Materials and methods}

Study population

After Institutional Review Board (IRB) approval, the University of Pittsburgh Center for Cranial Base Surgery database was queried to identify all cases of CSF rhinorrhea from Jan 1999-Aug 2015.

Inclusion criteria included adult and pediatric patients that underwent endoscopic repair of their CSF leaks by our multidisciplinary cranial base team, following ESS by otolaryngologists both internal and external to our institution. The exclusion criteria included endonasal surgery for the management of benign or malignant sinonasal/skull base tumors and those with a history of previous endoscopic skull base surgery.

The objective of this study was to describe the neurologic sequelae of skull base injury from ESS based on the latency to diagnosis. The cohort was divided into two groups based on the timing of the diagnosis of iatrogenic CSF leak with an early diagnosis group defined as less than 72 hours from the original procedure and a late group as greater than 72 hours. A retrospective review was conducted and included patient demographics, indication for surgery, history of previous ESS, imaging results, date of presentation, neurologic complications, beta-2- transferrin testing, anatomic site and size of skull base defect, method of repair, and use of perioperative CSF diversion with lumbar drainage. Pre-operative imaging was analyzed and the classification system developed by Keros was used to stage olfactory fossa anatomy ${ }^{(29)}$. To further characterize the preoperative skull base anatomy, we recorded the angle of the skull base relative line parallel to the hard palate. Both have been previously found to correlate with iatrogenic injury during ESS ${ }^{(12)}$. Data was collected through independent review of the electronic medical record.

The primary outcome measure was the incidence of neurologic complications, including postoperative meningitis, intracranial vascular injury, subarachnoid hemorrhage, frontal lobe injury, cerebrovascular accident (CVA), and pneumocephalus.

\section{Statistical analysis}

Descriptive statistics of all study variables were generated with the assistance of commercial statistical software (SPSS Version 20.0, IBM SPSS Inc, Chicago, IL, USA). Student t-test and fisher exact tests were used for continuous and categorical variables, respectively. All statistical tests were 2 -sided and $p$ values were evaluated such that consideration for statistical significance was $\mathrm{p}<0.05$.

\section{Results}

Seventeen patients were treated at our institution for iatrogenic skull base injuries resulting from ESS. Basic demographics can be found in Table 1. The mean age in our study group was 53 years (range 16-76) and 64.7\% (11/17) were female. Median follow up for the entire cohort was 9 months.

Injury occurred during standard ESS in 88\% (15/17) of cases and balloon sinuplasty in $12 \%(2 / 17)$. The primary surgical indications included the following: CRS 35.3\% (6/17), CRS with polyposis $52.9 \%(9 / 17)$, sphenoid sinus mucocele $5.9 \%(1 / 17)$, and frontal sinus mucocele $5.9 \%$ (1/17). Only $17.6 \%$ (3/17) had prior ESS. Symptoms upon presentation to our institution included clear rhinorrhea $(70.5 \%, 12 / 17)$, headache $(23.5 \%, 4 / 17)$, and altered mental status $(11.7 \%, 2 / 17)$. The mean length of hospital stay in our cohort was 4.9 days (Range 1-14).

Skull base injury was recognized intraoperatively only in $17.6 \%$ (3/17) of cases. Late diagnosis (> $72 \mathrm{hrs)} \mathrm{occurred} \mathrm{in} 64.7 \%$ $(11 / 17)$ of cases. Median time from surgery to the diagnosis of CSF leak was 7 days (range 0-56). The primary surgery was performed at a community hospital in $82.3 \%$ (14/17) of cases; $78.5 \%(11 / 14)$ of these were referred late ( $>72$ hours). Mean time to diagnosis of CSF fistula was reduced in those undergoing ESS at our institution vs. the community setting ( 1.0 day vs 12.3 days, $\mathrm{p}=0.33$ ) but this was not statistically significant. There was no association between late diagnosis and duration of hospital stay $(p=0.7)$.

Testing of nasal drainage for beta-2 transferrin was performed in 11 cases. This was positive in $72 \%(8 / 11)$ of cases with a surgically confirmed CSF leak.

Preoperative and postoperative imaging was reviewed for all patients. The Keros classification among our cohort was distributed as follows: Keros 1 (11.7\%, 2/17), Keros 2 (47.0\%, 8/17), Keros $3(41.1 \%, 7 / 17)$. The average olfactory fossa depth was $6.2 \mathrm{~mm}$. The average skull base angle was $14.8^{\circ}$.

No patient had placement of a lumbar spinal drain as primary treatment for the iatrogenic CSF leak. All patients underwent 
Table 1. latrogenic skull base injury during ESS: patient characteristics.

\begin{tabular}{|c|c|c|}
\hline \multirow[b]{2}{*}{ Patient variables } & \multicolumn{2}{|c|}{ Total patients $(n=17)$} \\
\hline & Number & $\%$ \\
\hline Age (Years), Median, Range & 53 & $16-76$ \\
\hline Follow Up (Months), Median & 9 & \\
\hline \multicolumn{3}{|l|}{ Sex } \\
\hline Male & 6 & $35.3 \%$ \\
\hline Female & 11 & $64.7 \%$ \\
\hline \multicolumn{3}{|l|}{ Procedure } \\
\hline FESS & 15 & $88.2 \%$ \\
\hline Balloon Sinuplasty & 2 & $11.8 \%$ \\
\hline \multicolumn{3}{|l|}{ FESS Indication } \\
\hline CRS & 6 & $35.3 \%$ \\
\hline CRS w Polyps & 9 & $52.9 \%$ \\
\hline Sphenoid Mucocele & 1 & $5.9 \%$ \\
\hline Frontal Mucocele & 1 & $5.9 \%$ \\
\hline \multicolumn{3}{|l|}{ Primary vs Revision } \\
\hline Primary & 14 & $82.4 \%$ \\
\hline Revision & 3 & $17.6 \%$ \\
\hline \multicolumn{3}{|l|}{ Preoperative Imaging } \\
\hline Mean Olfactory Fossa Depth (mm) & 6.2 & - \\
\hline Mean Skull Base Angle & 14.8 & - \\
\hline \multicolumn{3}{|l|}{ Time of Diagnosis } \\
\hline$<72$ Hours (Early) & 6 & $35.3 \%$ \\
\hline$>72$ Hours (Late) & 11 & $64.7 \%$ \\
\hline \multicolumn{3}{|l|}{ Location of Defect } \\
\hline Cribriform Plate & 10 & $58.8 \%$ \\
\hline Anterior Ethmoid Roof & 4 & $23.5 \%$ \\
\hline Posterior Ethmoid Roof & 1 & $5.9 \%$ \\
\hline Sphenoid & 1 & $5.9 \%$ \\
\hline Frontal Recess & 1 & $5.9 \%$ \\
\hline
\end{tabular}

successful endoscopic repair of their CSF leak. At the time surgery, the average defect size was $4.5 \mathrm{~mm}$. The description of the injury provided in the operative report was that of a "perforation" in 94\% (16/17) and a "crack" in 5.9\% (1/17). The injury was at the cribriform plate in $58.8 \%(10 / 17)$, anterior ethmoid roof in $23.5 \%$ (4/17), posterior ethmoid roof in 5.9\% (1/17), the sphenoid in 5.9\% (1/17), and frontal recess in 5.9\% (1/17). Most patients had an overlay (9/17) or multilayer (8/17) skull base reconstruction. A vascularized nasoseptal flap was used in (7/17) patients and a free mucosal graft was used in (7/17). Other graft material choices included banked pericardium (2/17) and Alloderm (1/17). A lumbar drain was used for an average duration of 5 days in $23.5 \%$ (4/17) of patients. Two patients had failed primary repair and underwent revision surgery. In both cases

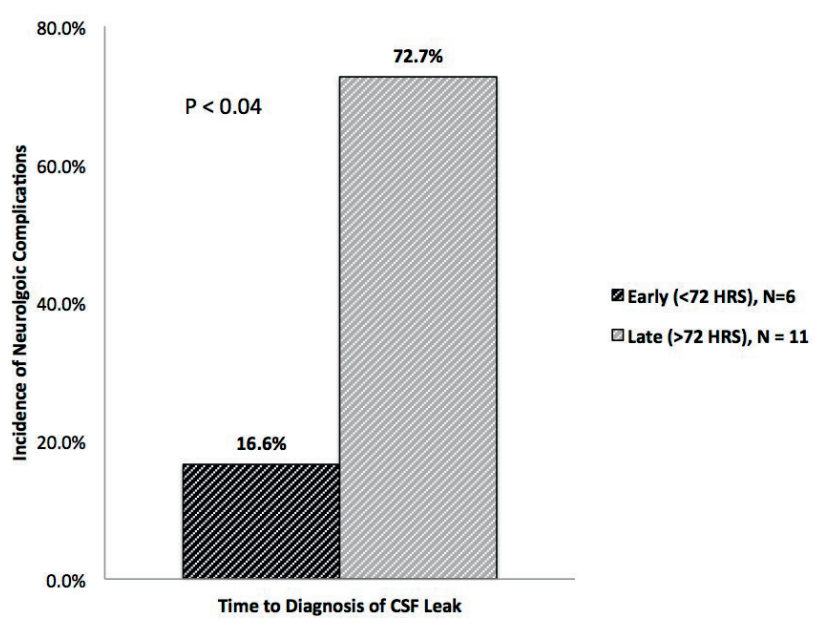

Figure 1. Incidence of neurologic complications vs time to diagnosis of CSF leaks after ESS.

the grafting material used initially was banked pericardium and revision entailed nasoseptal flap closure with lumbar drainage. The usage of pericardium as a graft material was used only early in our clinical experience and has since been abandoned.

Overall, $52.9 \%$ (9/17) of patients sustained a neurologic complications. Specifically, 47.1\% (8/17) developed postoperative ascending meningitis, $11.7 \%$ (2/17) developed pneumocephalus, $11.7 \%(2 / 17)$ sustained subarachnoid hemorrhage (SAH), and $5.8 \%(1 / 17)$ developed a frontal lobe injury. One patient developed a right parietal cerebrovascular accident (CVA) attributed to vasospasm from SAH (Table 2). All patients that experienced neurologic complications had undergone surgery at an outside community hospital. Time to diagnosis was related to the development of postoperative neurologic complications $(p<0.04)$ (Figure 1). Most significantly, the incidence of postoperative meningitis in those diagnosed in the late and early setting was $72.7 \%(8 / 11)$ and $0 \%(0 / 6)$ respectively $(p<0.01)$. Of note, no neurologic complications were seen in those diagnosed intraoperative. No patients developed persistent hydrocephalus as a consequence of meningitis.

With respect to permanent neurologic sequelae, 23.5\% (4/17) had persistent anosmia on follow up and one patient had new onset neuropsychiatric symptoms associated with a persistent frontal lobe abnormality on MRI.

Two patients in our cohort sustained skull base injury with resultant CSF leak following frontal balloon sinuplasty. One of these patients had significant pneumocephalus and diffuse subarachnoid hemorrhage on imaging. The site of injury in these two patients was the cribriform plate and anterior ethmoid roof. 
Table 2. Complications of skull base injury during ESS vs. time of diagnosis.

\begin{tabular}{|c|c|c|c|c|c|}
\hline \multirow[b]{2}{*}{ Neurologic Complications } & \multicolumn{2}{|c|}{ Early Diagnosis < 72 HRS (N=6) } & \multicolumn{2}{|c|}{ Late Diagnosis > 72 HRS (N=11) } & \multirow[b]{2}{*}{ P Value } \\
\hline & Number & $\%$ & Number & $\%$ & \\
\hline Neurologic Complication & 1 & 16.6 & 8 & 72.7 & $p<0.04$ \\
\hline Meningitis & 0 & 0.0 & 8 & 72.7 & $p<0.01$ \\
\hline Pneumocephalus & 1 & 16.6 & 1 & 9.0 & \\
\hline Subarachnoid Hemorrhage & 1 & 16.6 & 1 & 9.0 & \\
\hline Frontal Lobe Injury & 0 & 0.0 & 1 & 9.0 & \\
\hline CVA & 1 & 16.6 & 0 & 0.0 & \\
\hline
\end{tabular}
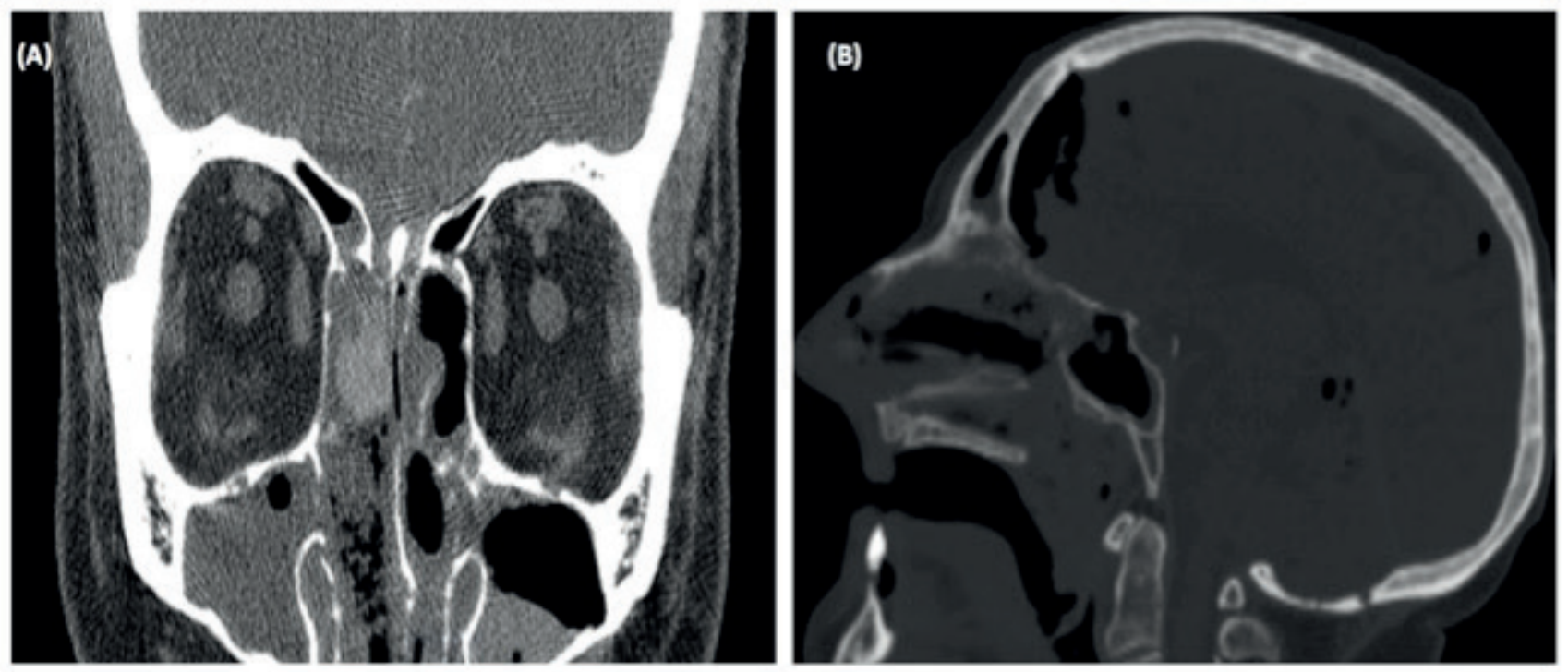

Figure 2. Radiographic examples of skull base injury after frontal sinus balloon sinuplasty. (A) CT Cisternogram demonstrating CSF fistula at the lateral lamella of the right cribriform plate. (B) Maxillofacial CT demonstrating an ethmoid roof skull base defect with associated pneumocephalus.

\section{Discussion}

Skull base injury during ESS is a rare but potentially catastrophic complication, occurring in $0.13-0.5 \%$ of cases ${ }^{(3-7)}$. Injury to intracranial structures, intracranial hemorrhage, pneumocephalus, and ascending infection with resultant meningitis or intraparenchymal brain abscess have all been reported ${ }^{(3,4)}$. Not surprisingly, skull base injury is also the most common cause for malpractice litigation after ESS ${ }^{(30)}$. Prior case reports have suggested that the morbidity associated with skull base transgression during ESS may be related to delay in diagnosis ${ }^{(31)}$.

In the current investigation, delays in the recognition of skull base injury and CSF rhinorrhea during sinus surgery were associated with increased neurologic complications and, specifically, significantly increased rates of postoperative meningitis $(72.7 \%$ vs $0 \%, p<0.04)$.

Postoperative meningitis resulting from skull base injury and resulting ascending infection is a significant source of morbidity. In the literature, the risk of death with acute bacterial meningitis in adults can be as high as $18-37 \%{ }^{(32)}$. In survivors, the incidence of long-term neurologic sequelae can be as high as $30 \%{ }^{(32)}$.

The incidence of meningitis in the setting of CSF rhinorrhea has been cited to be $10-19 \%{ }^{(15,28)}$ and may increase with time ${ }^{(33)}$. Fortunately, endoscopic repair of skull base defects in the early postoperative setting has been associated with a reduction in the risk of meningitis to $0.3 \%$ annually in one large metaanalysis ${ }^{(17)}$.

In our study, $64.7 \%$ of CSF leaks were diagnosed late, with the majority occurring in primary surgeries (83\%) and in the community setting (83\%). This suggests that skull base injury during ESS is not simply an entity seen in high risk patients treated with revision surgery at tertiary academic referral centers. Our data suggest that CSF leaks are often not recognized during primary surgery for CRS, leading to significant morbidity. Failure to 
recognize a CSF leak at the time of injury may reflect the training and knowledge of the surgeon, poor visualization due to bleeding or pathology, or the size of the defect.

Interestingly, two patients sustained skull base injuries from balloon sinuplasty. Balloon sinuplasty was FDA approved in 2005 and has since gained popularity as a minimally invasive method of dilating sinus outflow tracts. Proponents of balloon sinuplasty cite its' safety and minimally invasive nature as one of the primary advantages. In the largest prospective study by Bolger at al, there were no serious adverse events or CSF leaks reported in 115 patients ${ }^{(34)}$. Only one other report of skull base injury as a result of balloon sinuplasty exists currently in the literature ${ }^{(35)}$. Our findings underline the fact that the potential for skull base injury exists during surgical management of CRS regardless of the type of instumentation used.

The incidence of a Keros type 3 classification (olfactory fossa depth $>7 \mathrm{~mm}$ ) in our cohort was $41.1 \%$ compared to the incidence described by Keros in his initial studies (18\%) ${ }^{(29)}$. This is consistent with previous reports describing that increased depth of the olfactory fossa poses a risk factor for iatrogenic CSF leak ${ }^{(12-13)}$.

The average skull base defect size in this study was $4.5 \mathrm{~mm}$ and in $94 \%$ of cases this defect was described as a "perforation" as opposed to a "crack". This may provide some insight into the mechanism of these skull base injuries during. Our results would suggest that these injuries result from an instrument directly transgressing the skull base as opposed to excessive torque applied to a bony structure in continuity with skull base above. This is primarily speculative and would need to be evaluated in a larger study.

As with most studies on complications of ESS, sample size was a limitation of this study as was its' retrospective design. In the future, a larger, prospective study would be advisable to further delineate the importance of timely diagnosis in managing complications after ESS.

\section{Conclusion}

Skull base injury is an uncommon but dangerous complication of ESS. It can occur with traditional instrumentation as well as balloon sinuplasty. This study suggests that the delayed diagnosis of iatrogenic skull base injuries is common and associated with high rates of neurologic morbidity and postoperative meningitis. A sound understanding of risk factors, sinonasal anatomy, the clinical presentation, and surgical management of iatrogenic skull base injuries is vital for today's practicing sinus surgeon. A high index of suspicion for CSF rhinorrhea should be maintained in the intraoperative and postoperative setting after ESS despite the rarity of this complication.

\section{Authorship contribution}

MK: Design, data collection, data analysis, composed manuscript; EW: design, data analysis, manuscript revision; CS, SL: Manuscript review.

\section{Conflict of interest}

There are no conflicts of interest present.

\section{References}

1. Hamilos DL. Chronic Rhinosinusitis: epidemiology and medical management. J Allergy Clin Immunol 2011; 128(4): 693-707.

2. Bhattacharyya N. Ambulatory sinus and nasal surgery in the United States: Demographics and perioperative out comes. Laryngoscope 2010; 120 (3): 635638.

3. Krings JG, Kallogjeri D, Wineland A et al. Complications of Primary and Revision Functional Endoscopic Sinus Surgery for Chronic Rhinosinusitis. Laryngoscope 2013; 124:838-845

4. Stankiewicz JA, Lal D, Connor M. Complications in Endoscopic Sinus Surgery for Chronic Rhinosinusitis: A 25 Year Experience. Laryngoscope 2011; 121: 26842690

5. Hopkins C, Slack R, Lund V. Long-term outcomes from the English national comparative audit of surgery for nasal polyposis and chronic rhinosinusitis. Laryngoscope 2009; 119: 2459-65.

6. Ramakrishnan $\mathrm{V}$, Kingdom T, Nayak J, et al.
Nationwide incidence of major complications in endoscopic sinus surgery. Int Forum Allergy Rhinol 2012; 2:34-39.

7. May M, Levine $H$, Mester $S$, et al. Complications of endoscopic sinus surgery: analysis of 2108 patients-incidence and prevention. Laryngoscope 1994; 104:10801083.

8. Stankiewicz J. Complications of endoscopic intranasal ethmoidectomy. Laryngoscope 1987; 97:1270-1273.

9. May M, Levine $H$, Schaitkin B. Complication of endoscopic sinus surgery: a meta-analysis. Laryngoscope 1994; 104: 1081-1084.

10. Castillo L, Verschuur HP, Poissonnet G, et al. Complications of endoscopically guided sinus surgery. Am J Rhinol 2005; 19:274-281.

11. Keerl R, Stankiewicz J, Weber R, et al. Surgical experience and complications during endonasal sinus surgery. Laryngoscope 1999; 109:546-550.

12. Heaton CM, Goldberg AN, Pletcher SD, et al. Sinus Anatomy Associated with Inadvertent Cerebrospinal Fluid Leak During Functional Endoscopic Sinus Surgery. Laryngoscope
2012; 122:1446-1449.

13. Lee JC, Song YJ, Chung YS, et al. Height and shape of the skull base as risk factors for skull base penetration during endoscopic sinus surgery. Ann Otol Rhinol Laryngol 2007; 116: 199-205.

14. Grevers G. Anterior skull base trauma during endoscopic sinus surgery for nasal polyposis: preferred sites for iatrogenic injuries. Rhinology 2001; 39: 1-4.

15. Daudia A, Biswas D, Jones NS. Risk of meningitis with cerebrospinal fluid rhinorrhea. Ann Oto Rhinol Laryngol 2007; 116(12): 902-905.

16. Dandy WD. Pneumocephalus (intracranial pneumocele or aerocele). Arch Surg 1926; 12:949-982.

17. Hegazy HM, Carrau RL, Synderman $\mathrm{CH}$, et al. Transnasal endoscpic repair of cerebrospinal fluid rhinorrhea: a meta analysis. Laryngoscope 2000; 110:1166-1172.

18. Zweig J, Carrau R, Celin S et al. Endoscopic Repair of cerebrospinal fluid leaks to the sinonasal tract. Predictors of success. Otolaryngol Head Neck Surg. 2000; 123; 
195-200.

19. Snyderman $\mathrm{CH}$, Kassam AB, Carrau R, et al. Endoscopic reconstruction cranial base defects following endonasal skull base surgery. Skull Base 2007; 17: 73-78.

20. Wigand ME. Transnasal ethmoidectomy under endoscopical control. Rhinology 1981; 19: 17-20.

21. MCMains KC, Gross CW, Kountakis SE. Endoscopic Management of Cerebrospinal Fluid Rhinorrhea. Laryngoscope 2004; 114: 1833-1836.

22. Wheless SA, McKinney KA, Carrau RL, et al. Nasoseptal Flap Closure of Traumatic Cerebrospinal Fluid Leaks. Skull Base 2011; 21 (2) 93-97.

23. Platt M, Shaye D, Parnes S. Management of unexpected CSF fistula during endoscopic sinus surgery. Curr Opin Otolaryngol Head Neck Surg 2009; 17: 28-32.

24. Hou W, Wen Y, Wang Z. Clinical Analysis of the cranial complications of endoscopic sinus surgery. Acta Oto-Laryngologica 2013; 133: 739-743.

25. Bumm K, Heupel J, Bozzato, et al. Localization and inflication pattern of iatrogenic skull base defects following endoscopic sinus surgery at a teaching hospital. Auris Nasus Larynx 2009; 36:671-676.

26. Soudry E, Turner JH, Nayak JV. Endoscopic
Reconstruction of Surgically Created Skull Base Defects: A Systematic Review. Otolaryngol Head Neck Surg. 2014; 150 (5) 730-738.

27. Senior BA, Jafri K, Benninger M. Safety and efficacy of endoscopic repair of CSF leaks and encephaloceles: a survey of the members of the American Rhinologic Society. Am J Rhinol. 2001; 15: 21-25.

28. Schoentgen C, Henaux P, Godey B, et al. Management of post-traumatic cerbrospinal fluid (CSF) leaks of the anterior skull base. 10 year experience. Acta Otolaryngol 2013; 133(9) 944-950.

29. Keros P (1965). Uber die praktische Bedeutung der Niveauunterschiede der Lamina cribrosa des Ethmoid. Z Laryngol Rhinol 41, 808-809.

30. Lynn-Macrae AG, Lynn Macrae RA, Emani J, et al. Medicolegal analysis of injury during endoscopic sinus surgery. Laryngoscope 2004; 114: 1492-1495.

31. Hassab MH, Eweiss AZ, Ibrahim AA. Missed skull base injury during sinonasal surgery: A dangerous scenario still existing. Ear Nose Throat J 2015; 94 (8) 37-42.

32. van de Beek D, Gans J, Tunkel A, et al. Community Acquired Bacterial Meningitis in Adults. New Engl J Med. 2006; 354(1) 44-53.
33. Eljamel MS, Foy PM. Acute traumatic CSF fistula: the risk of intracranial infection. $\mathrm{Br}$ Neurosurg 1990; 4(5): 381-5.

34. Bolger WE, Brown $\mathrm{CL}$, Church $\mathrm{CA}$, et al Safety and outcomes of balloon catheter sinusotomy: A multicenter 24 week analysis in 115 patients. Otolaryngol Head Neck Surg. 2007; 137: 10-20.

35. Tomazic PV, Stammberger $H$, Koele W, et al. Ethmoid roof CSF - leak following frontal sinus balloon sinuplasty. Rhinology 2010; 48 (2): 247-50.

\section{Mark Kubik, MD}

Center for Cranial Base Surgery

Department of Otolaryngology

200 Lothrop Street, EEI 521

Pittsburgh PA 15213

USA

Tel: +1-412-864-1744

Fax: +1-412-647-2080

E-mail: kubikmw@upmc.edu

ADVERTISEMENT

18th International Course on

Reconstructive and Aesthetic Surgery of the Nose and Face

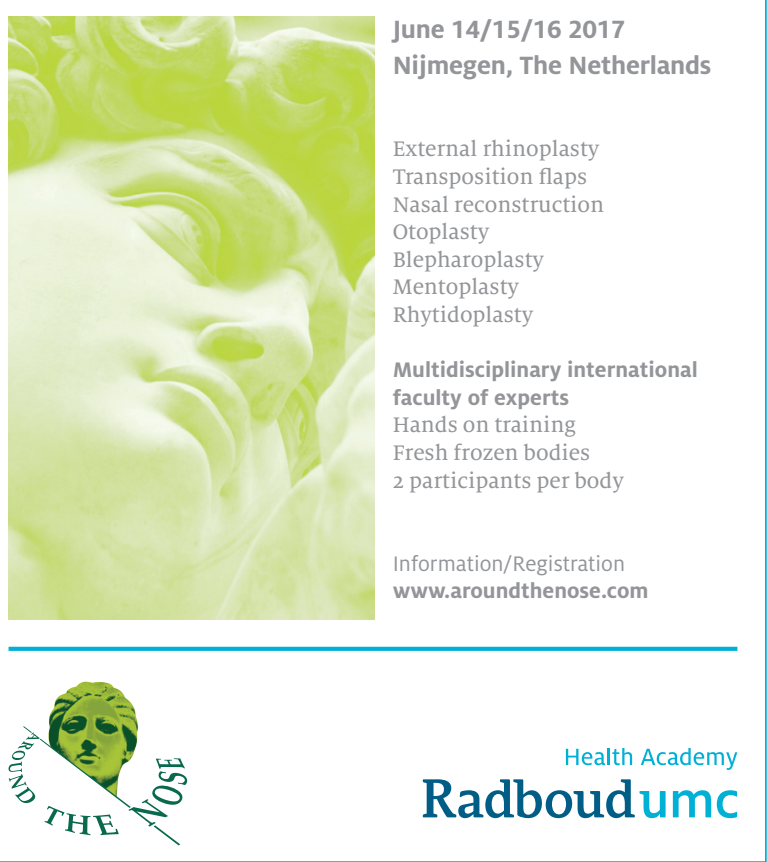

